Como citar este artículo:

López, J.A. (2020). Política de la información, las ONG y los medios de comunicación en

los conflictos por derechos humanos: un balance. Revista Eleuthera, 22 (1), 88-105. DOI:

10.17151/eleu.2019.20.6.

\title{
POLÍTICA DE LA INFORMACIÓN, LAS ONG Y LOS MEDIOS DE COMUNICACIÓN EN LOS CONFLICTOS POR DERECHOS HUMANOS: UN BALANCE*
}

\author{
INFORMATION POLICY, NGOs AND MASS MEDIA IN HUMAN RIGHTS \\ CONFLICTS: AN ASSESSMENT
}

JAIRO ANTONIO-LÓPEZ**

\section{Resumen}

Objetivo. El presente artículo presenta una revisión crítica de la literatura académica que estudia la relación entre las ONG y los medios de comunicación en los conflictos por derechos humanos. Metodología. A partir de una revisión documental, a manera de balance de estado del arte, se clasifican y analizan los principales enfoques y tesis de la literatura. Resultados. Se argumenta que la literatura académica ha enfatizado en cómo la política de la información se constituye en un repertorio modular del activismo en defensa de los derechos humanos, pero mantiene un sesgo en el análisis de las dinámicas, organizaciones y medios internacionales. Conclusiones. Se plantea la necesidad de desarrollar una agenda de investigación que tenga en cuenta los procesos y relaciones entre las ONG y los medios de comunicación a nivel estatal, además de repensar dicha relación en contextos de grave violencia contra periodistas con el fin de explicar las posibilidades y límites de la política de la información.

Palabras clave: ONG, derechos humanos, política de la información, medios de comunicación.

\begin{abstract}
Objective. This paper presents a critical review of the academic literature that studies the relationship between NGOs and mass media in human rights conflicts. Methodology. From a documentary review, as an assessment of the state of the art, the main approaches and theses of the literature are classified and analyzed. Results. It is argued that the academic literature has emphasized how information policy constitutes a modular repertoire of activism in defense of human rights, but maintains a bias in the analysis of international dynamics, organizations and mass media. Conclusions. There is a need to develop a research agenda that takes into account the processes and relationships between NGOs and mass media at the state level, in addition to rethinking this relationship in contexts of serious violence against journalists, in order to explain the possibilities and limits of the information policy.
\end{abstract}

Key words: NGOs, human rights, information policy, media.

\footnotetext{
*Artículo de revisión, parte del proyecto de investigación "Medios de comunicación, poder e información en los conflictos por derechos humanos".

${ }^{* * *}$ Universidad Veracruzana. Veracruz, México. E-mail: jairolopez32@gmail.com.

(D) orcid.org/0000-0003-4877-6708 Google Scholar
} 


\section{Introducción}

El mundo en general, y América Latina en particular, atraviesa momentos críticos en relación con el compromiso de los gobiernos con el respeto de los derechos humanos. Una de las manifestaciones más claras de esta situación son las políticas y prácticas gubernamentales (tanto en los países centrales como periféricos) de restricción o confrontación a la libertad de expresión y de prensa, al mismo tiempo que un aumento de la violencia contra el periodismo crítico ${ }^{1}$. Por otro lado, existe una tendencia global de los gobiernos por restringir el rol de los activistas y organizaciones no gubernamentales de derechos humanos, dado su creciente esfuerzo por documentar, analizar y visibilizar las violaciones de derechos humanos y el abuso de poder (Dupuy, Ron \& Prakash, 2016).

Además de lo anterior, en varios países de América Latina se presentan graves escenarios de violencia e implementación de políticas de militarización con terribles consecuencias para los derechos humanos. Precisamente las investigaciones académicas han encontrado que los y las activistas de derechos humanos encuentran grandes límites para difundir sus demandas en contextos de implementación de políticas de seguridad y militarización bajo estrategias de "combate al terrorismo", "lucha contra el crimen organizado", o "amenaza a la seguridad nacional”. Esto se debe, entre otras razones, al respaldo que este tipo de políticas pueden tener por parte de la población, la capacidad de los gobiernos de generar discursos retóricos para presentarse como defensores de derechos humanos ante la "amenaza a la seguridad”, así como por la dificultad que encuentran las redes, organizaciones, movimientos y medios para producir "presión creíble" dados los "difusos responsables de las violaciones" (Brysk, 2013, 2009; Brysk \& Shafir, 2007; Cárdenas, 2010, 2007; Dancy \& Sikkink, 2017; Gordon, 2014; Jetschke, 2010; Jetschke \& Liese, 2013; Shor, 2008).

Frente a estas restricciones, no todas las violaciones a los derechos humanos tienen repercusión pública y muchos medios de comunicación no siempre tienen líneas editoriales claramente vinculadas con la denuncia en materia de derechos humanos. Por estas razones, la relación entre organizaciones no gubernamentales, activistas de derechos humanos y medios de comunicación toma relevancia; no obstante, en América Latina existen pocas investigaciones que la aborden como parte de las estrategias de defensa de los derechos humanos y fortalecimiento de la circulación de la información en la materia.

En tal sentido, el objetivo del presente artículo es realizar un balance crítico de las principales líneas de investigación, ejes y preguntas de la literatura que estudia la relación entre las ONG de derechos humanos y medios de comunicación. El argumento principal es que en

\footnotetext{
${ }^{1}$ Según los informes mundiales, en los últimos años ha habido una caída constante de los indicadores de libertad de expresión, de prensa y democracia. Contrario a lo esperado con la cada vez mayor firma y ratificación de tratados, convenios y convenciones de derechos humanos, el mundo experimenta significativos retrocesos de libertades fundamentales (Freedom House, 2018).
} 
Política de la información, las ong y los medios de comunicación en los conflictos por derechos humanos: un balance

los estudios especializados predomina el análisis de la política de información como estrategia del activismo en derechos humanos, con dos énfasis: primero, la capacidad que puedan tener las ONG de incidir en el contenido de los medios de comunicación, definidos estos como mediadores principales de las demandas colectivas; y segundo, el análisis del rol que tienen las ONG como productoras autónomas de información y los beneficios o costos que esto puede tener para los medios de comunicación. Ambos énfasis reproducen un sesgo internacionalista de las preguntas sobre la relación entre ONG de derechos humanos y medios de comunicación, que no ha permitido abordar y explicar las dinámicas que posibilitan o limitan la circulación e interacción de información entre ONG de derechos humanos y medios de comunicación en contextos estatales.

Primero, defino analíticamente la política de la información como repertorio establecido en todo el mundo para la defensa de los derechos humanos, delimitando la importancia que toma la relación entre ONG y medios de comunicación en dicha dinámica. Segundo, expongo críticamente los enfoques de la literatura en torno a esta relación, distinguiendo el énfasis dado al rol de los medios como mediadores y el de las ONG como productoras novedosas de información. Finalmente, planteo un cuestionamiento al sesgo en el análisis de la dimensión internacional y las grandes organizaciones norteamericanas, exponiendo la necesidad de retomar los aportes de la literatura para profundizar en el estudio de este tipo de procesos dentro de los Estados.

\section{Discusión}

\section{La política de la información: un repertorio establecido en los conflictos por derechos humanos}

Como plantea Hincapié $(2017,2015)$, los derechos humanos son principios éticos, dispositivos jurídicos y recursos de movilización para la acción colectiva. Especialmente desde la expansión del régimen internacional de los derechos humanos en los años setenta del siglo XX, los movimientos sociales, organizaciones no gubernamentales y actores colectivos han adelantado estrategias y repertorios para la defensa y socialización de los derechos humanos en escenarios de abusos del poder (López, 2017; Risse, Ropp \& Sikkink, 2013; Simmons, 2009; Tsutsui, Whitlinger \& Lim, 2012; Wotipka \& Tstutsui, 2008).

En este proceso, se ha denominado como “política de la información” (Keck \& Sikkink, 2000) a las estrategias de documentación, investigación y denuncia pública que llevan a cabo las redes y activistas de derechos humanos para generar presión sobre los gobiernos en contextos de violencia y violaciones de derechos humanos. Dentro de las muchas actividades que desarrollan los actores colectivos como parte de su política de información se encuentra documentar sistemáticamente violaciones de derechos humanos, realizar análisis de contextos y causas de 
las violaciones, publicar informes con base en datos y testimonios, generar campañas que den visibilidad pública por medio de casos emblemáticos, cabildear frente a actores poderosos y órganos intergubernamentales, entre muchas otras (Brysk, 1994; Burgerman, 2001; Keck \& Sikkink, 2000; Risse, Ropp \& Sikkink, 1999). Como recuerda Brysk (2013), los hechos y las violaciones de derechos humanos no hablan solas, éstas se conocen y difunden a partir de estrategias de comunicación, narración y socialización que implican decisiones, habilidades y capacidades de actores concretos. Igualmente, la forma como los hechos relacionados con derechos humanos son narrados tienen implicaciones sustanciales, tanto en la construcción de dicha realidad como en la posibilidad de generar empatía y solidaridad para que las cosas cambien (Borer, 2012; Brysk, 2013).

La política de información es una estrategia colectiva muy generalizada en la defensa de los derechos humanos por tres razones, todas relacionadas con los recursos con los que cuentan los activistas y organizaciones no gubernamentales. Primero, está basada en el establecimiento de estándares altamente aceptados a través de la Declaración Universal de los Derechos Humanos y los diferentes tratados regionales e internacionales, es decir, cuando se denuncia la "perversidad" de una práctica se hace con base en principios moralmente aceptados. Segundo, se ha consolidado una amplia variedad de ONG, organizaciones intergubernamentales y algunas organizaciones gubernamentales, dedicadas a investigar con sistematicidad los abusos en materia de derechos humanos, consolidando una reputación positiva que ha permitido que su información sea tomada con mucha seriedad. Tercero, hasta el momento dentro del campo de los derechos humanos los actores civiles no tienen muchos otros medios de presión para generar cambios, razón por la cual esta estrategia comúnmente está dirigida a ejercer presión sobre actores con capacidad coercitiva, como pueden ser cortes y gobiernos poderosos, para que tomen medidas de castigo activando indirectamente mecanismos de rendición de cuentas (Cárdenas, 2007; Franklin, 2015; Hafner-Burton, 2008; Hagan, 2010; Keck \& Sikkink, 2000; Murdie \& Davis, 2011; Tarrow, 2005).

En el centro de la apuesta por generar información por parte de activistas de derechos humanos está el supuesto según el cual "nombrar y avergonzar" (naming and shaming) puede ser efectivo en la medida que los Estados tienen interés en mantener una correcta "reputación” en el escenario internacional. En otras palabras, la política de la información tiene en su origen la idea según la cual los derechos humanos son principios éticos y morales frente a los cuales los gobiernos no pueden presentar rechazo (la ola de regresiones autoritarias y anti-derechos humanos que vivimos hoy parece ir en contra de estas ideas). En este sentido, cuando se visibilizan públicamente practicas indeseadas como las violaciones a los derechos humanos, los Estados verían, en teoría, afectados sus intereses y tendrían incentivos para cambiar su comportamiento, o por lo menos, actores poderosos se verían llamados a presionar sobre éstos (Cárdenas, 2007; Keck \& Sikkink, 2000; López, 2013; Risse et al., 2013). 
Política de la información, las ong y los medios de comunicación en los conflictos por derechos humanos: un balance

Los estudios clásicos como los de Keck \& Sikkink (2000) y Brysk (1994) habían definido a las redes de activismo (de las cuales la política de la información en derechos humanos es su principal repertorio de movilización) como espacios de horizontalidad, donde los actores involucrados tienen relaciones de cooperación ${ }^{2}$. Sin embargo, se han elaborado críticas certeras a esta lectura en la medida que la capacidad de generar información es un recurso especial para el cual se requieren habilidades y capacidades económicas, de infraestructura, de personal y conocimiento (López e Hincapié, 2015). En otras palabras, no todos los actores colectivos tienen habilidades desarrolladas para generar información, contratar personal profesional, tener vínculos en el campo y, al mismo tiempo, establecer interlocución con actores gubernamentales e internacionales. Autores como Bob (2010, 2005), Gordon (2008) y López e Hincapié (2015), han señalado las relaciones de poder, interés y conflicto que se generan entre las propias organizaciones no gubernamentales y los actores colectivos instituidos en la defensa de los derechos humanos ${ }^{3}$. Igualmente, la capacidad que tienen las organizaciones para que su información circule y se posicione en espacios estratégicos como los medios de comunicación varía sustancialmente de una a otra (no es lo mismo una organización posicionada de la capital de un país y una organización de las regiones del mismo país).

Pruce y Cosima (2016) proponen una interesante tipología para distinguir los niveles y ámbitos de influencia de la política de la información que llevan a cabo los y las activistas en general, y las ONG en particular. En primer lugar, cuando la política de información está orientada a la influencia en el mundo jurídico, ésta mantiene un tono claramente legal, delimitado estrictamente a "documentar", "recopilar evidencia" y "demostrar", siempre bajo una lógica científica y jurídica. En segundo lugar, cuando la información está orientada específicamente a "nombrar y avergonzar" frente al público estaríamos hablando de estrategias de información para "revelar", donde los medios de comunicación cobran un rol más importante dado que la apuesta se concentra en impulsar cambios con base en información, objetivo que sólo se puede lograr cuando ésta circula, es accesible y se hace visible para grandes públicos (incluidos los actores con influencia y poder de incidencia). Por último, el impulso del conocimiento sobre derechos humanos en audiencias globales, el cual está orientado ya no sólo a impactar en los medios para difundir información y evidencia, sino que ésta transcienda las fronteras comunicando y convenciendo a actores internacionales sobre la urgencia de actuar sobre la situación.

\footnotetext{
${ }^{2}$ Esta definición estaba muy vinculada a una idea original sobre "la sociedad civil" donde ésta era definida como una "esfera de acción” separada del estado y el mercado, con rasgos democráticos y de horizontalidad. Lo cierto es que dentro de la llamada sociedad civil existen diversas tendencias y orientaciones con valores liberales o conservadores, así como relaciones de interés y poder, que pueden reproducir relaciones verticales y elitistas (Olvera, 1999).

${ }^{3}$ Esta dinámica de producción de información y conocimiento se inscribe en la tensión señalada por Álvarez (2009) en relación con la "ongenización" de los movimientos sociales, es decir, a la marcada necesidad de generar procesos de profesionalización para vincularse y relacionarse de manera sostenida y eficiente con las instituciones gubernamentales. Este es un proceso que también ha sido explicado con claridad por Neil Stammers (2009), en relación con la necesidad que encuentran los movimientos sociales que promueven y defienden los derechos humanos de entablar relaciones estables con ONG profesionales, como grandes mediadoras entre los procesos locales y de base con los gobiernos y el Estado (López e Hincapié, 2015).
} 
De esta manera, dadas las particularidades de la política de la información en la defensa de los derechos humanos, la relación entre las demandas colectivas, las ONG y los medios de comunicación se vuelve central. Cuando los medios de comunicación informan e investigan sobre problemas puntuales, tienen la capacidad de producir cambios sobre la percepción social y posicionar debates en agendas más generales y públicas (Castells, 2011; Thompson, 2001). Así, cuando los movimientos y organizaciones no gubernamentales logran incidir en la generación de noticias que visibilicen los abusos del poder y las violaciones de derechos humanos, se pueden constituir como "contrapoderes", potenciando un efecto democratizador de la esfera pública y en la defensa de los derechos (Castells, 2011; Rosanvallon, 2007; Stammers, 2009; Tumber \& Waisbord, 2004; Waisbord, 2016).

\section{ONG y medios de comunicación: entre la mediación y la autoproducción}

Como se ha establecido hasta este punto, la política de la información como estrategia central en el activismo de defensa de los derechos humanos es el principal eje para entender la relación entre ONG y los medios de comunicación, sus potencialidades y limitaciones. Desde el origen del activismo global por los derechos humanos, en los años sesenta y setenta del siglo XX, se encuentra una estrecha relación entre las nacientes organizaciones no gubernamentales como Amnistía Internacional y Human RightsWatch con la prensa como plataforma de denuncia global, relación establecida principalmente en la defensa de los presos políticos de los regímenes autoritarios (Keck \& Sikkink, 2000; Menon, 2009; Risse et al., 1999).

En todos los casos, la principal vía de denuncia que seguían las organizaciones de derechos humanos era la de publicar notas en la prensa, donde documentaban, lo mejor que podían, los casos de violaciones de derechos humanos. Ahora bien, como claramente lo plantean Winston y Pollock (2016), en esa época:

Los periódicos se publicaban exclusivamente en papel impreso, las noticias de televisión se transmitían por radio y se recibían por antenas, y las cartas de activistas de derechos humanos dirigidas a funcionarios del gobierno se escribían en máquinas de escribir y se enviaban por correo. (p. 303)

En otras palabras, si bien desde el origen de las estrategias de activismo en defensa de los derechos humanos ha existido un vínculo estrecho entre ONG y medios de comunicación, hoy nos encontramos ante realidades tecnológicas, de capacidad de acción colectiva y circulación de información muy diferentes, razón por la cual es importante entender cómo se dan y estudian estas interacciones. 
Política de la información, las ong y los medios de comunicación en los conflictos por derechos humanos: un balance

\section{Los medios como mediadores de la política de la información}

El primer gran conjunto de investigaciones sobre la relación entre ONG de derechos humanos y medios de comunicación tienen en común el definir a los medios "como gatekeepers clave que deciden, según las normas de noticias establecidas, cuál de los problemas aparentemente infinitos de derechos humanos puede ocupar una parte de la agenda de noticias" (Powers, 2016a, p. 316). En otras palabras, estas investigaciones demuestran que los medios de comunicación no sólo son aliados estratégicos de las ONG y los activistas de derechos humanos, sino que tienen una agencia fundamental que los vuelve mediadores activos en la posibilidad de que las denuncias de derechos humanos circulen y tengan eco.

Esta mediación de los medios de comunicación en la política de la información es atendida desde diversos ángulos. En primer lugar, quienes estudian los casos en los que las ONG logran tener cobertura en los medios, destacando la forma como los actores colectivos construyen "la credibilidad" de la información y el drama que denuncian, con la finalidad de traducir los temas de derechos humanos a las normas dominantes de las noticias y medios que favorecen "el conflicto y el espectáculo" (Cottle \& Nolan, 2007; Fenton, 2010; Powers, 2016c; Waisbord, 2011). En este sentido, Waisbord (2011) señala que en América Latina las ONG siguen un “enfoque pragmático" en su relación con la prensa con el fin de lograr visibilidad, es decir, si bien muchos integrantes de las ONG tienen perspectivas críticas frente a los principales medios y la forma como suelen informar y cubrir problemas, éstos no entran en estrategias de confrontación entendiendo que su intermediación es muy importante para su trabajo.

En segundo lugar, se señala que uno de los factores fundamentales del impacto que pueden tener las denuncias de las ONG en los medios es el desarrollo de estrategias de "narración y reportaje del dolor", especialmente cuando éstas refuerzan representaciones de "la pobreza y los dramas humanitarios del sur en los medios del norte” (Dogra, 2014; Orgad, 2013; Rothmyer, 2011). Dentro de esta línea de análisis, Seaga (2012) plantea que el periodismo de derechos humanos, además de las limitaciones que encuentra por las políticas editoriales de informar con dramatismo sobre violaciones de derechos en sociedades no occidentales o no desarrolladas (a contraluz de lo que ocurre en las sociedades occidentales), enfrenta también la barrera de no atender las "formas indirectas de violencia estructural y cultural" que originan la violencia y violaciones, concentrándose en acontecimientos y situaciones de un momento. Esto se ve reforzado por lo que Thrall, Steculla y Sweet (2014) denominan como cortos ciclos de atención de los medios y la corta cobertura de los problemas que son capaces de realizar, razón por la cual la mayoría de los temas y problemas asociados con los derechos humanos, sus causas y razones, no logran recibir atención de los medios de comunicación.

Estos resultados son consistentes con aquellos estudios que han señalado que la mediación de los medios de comunicación está, generalmente, limitada a la cobertura y resonancia de 
violaciones de derechos civiles y políticos (por ejemplo, tortura, detención ilegal) (Clark, 2001; Hopgood, 2006; Powers, 2015; Winston, 2001). Además, la mayoría de los esfuerzos de generación de información y denuncias públicas de las grandes ONG se "desinflan” en la medida que hay temas vetados para la lógica de los medios y los intereses de los gobiernos centrales (Murdie \& David, 2011; Ron, Ramos y Rodgers, 2005).

En tercer lugar, dentro de la misma agenda que entiende a los medios de comunicación como mediadores de la política de información, autores críticos como Bob $(2005,2010)$ han señalado las "estrategias de marketing mediático" de las organizaciones de derechos humanos, es decir, la forma como ciertas ONG posicionan su "marca" en los medios y legitiman su prestigio (con casos como Amnistía Internacional o Greenpeace). En sus estudios, Bob ha resaltado la importancia de las estrategias de comunicación para las organizaciones, no sólo para difundir la información sobre las denuncias que realizan, sino también para posicionarse como actor visible, creíble y con prestigio en lo que denomina "el mercado de movimientos morales" (Bob, 2002). En este sentido, sus aportes son importantes para señalar que en la política de la información prevalecen los intereses y objetivos de pequeños grupos de ONG, por lo general de élites con capacidad de movilizar recursos, teniendo como efecto la exclusión de las coberturas de noticias sobre derechos humanos a otro tipo de organizaciones y actores con perfiles menos visibles.

Estos resultados, muy críticos con el rol de las grandes ONG norteamericanas, se encuentran en la misma línea de los trabajos realizados por Carpenter (2007a; 2007b), en la medida que identifican las disputas por "certificar los reclamos de derechos humanos" entre las ONG. Estas disputas de certificación afectan la forma y probabilidad de que ciertos temas y causas impulsadas por los activistas de derechos humanos sean retomadas o no en los medios, determinando la capacidad de que ciertas agendas (por ejemplo, la violencia contra niños y niñas y no temas sobre violencia contra migrantes) y áreas geográficas tengan visibilidad (Carpenter; 2014; 2007a).

Finalmente, en relación con las nuevas tecnologías los estudios señalan el esfuerzo de las ONG por aumentar el público receptor de los mensajes, de allí que algunas de las más importantes organizaciones internacionales han acudido a planes de publicidad donde incluyen producción muy elaborada de videos, campañas con personajes famosos e influencia (actores y actrices, deportistas, artistas), documentales, mensajes para recaudar fondos, entre otras (Pruce, 2015). Si bien las nuevas tecnologías para documentar y generar información sobre problemas relacionados con el respeto de los derechos humanos aumentan, estas estrategias no suplen las tradicionales prácticas de documentación y elaboración de informes detallados (Ristovska, 2016). Es decir, las nuevas tecnologías han sido herramientas para fortalecer las tradicionales prácticas de impulsar “campañas" por hacer pública y visible una situación, tratando de convencer a otros sobre la importancia de retomar y apoyar una causa (Becker, 2013; Nash, 2009). 
Política de la información, las ong y los medios de comunicación en los conflictos por derechos humanos: un balance

\section{Las ONG como productoras de información para los medios}

Además de la relación de los medios de comunicación como "mediadores" de la política de la información, los acelerados cambios tecnológicos han abierto un campo de práctica y reflexión sobre las nuevas estrategias para la producción de la propia información de derechos humanos. En este sentido, diversos trabajos señalan el nuevo rol de "informantes" que pueden tener los ciudadanos y ciudadanas activistas al tener disponibilidad de teléfonos móviles para registrar y circular casos de violaciones de derechos humanos (Becker, 2013; Bennett y Segerberg, 2012; Chadwick, 2013). Dados estos cambios, las ONG de derechos humanos han rediseñado estrategias para la circulación de información, incorporando "funciones aparentemente periodísticas" a sus propios cuerpos de trabajo. Así, como recuerda Powers (2016), organizaciones como Human Rights Watch y Amnistía Internacional ahora contratan fotógrafos y productores de video para acompañar los informes de investigación, así como han adelantado estrategias para ampliar sus sedes y presencia de campo directo en el "sur global".

La literatura que analiza estos procesos de fortalecimiento de las ONG como productoras directas de la información para los medios señala dos elementos importantes. Por un lado, la forma como las nuevas tecnologías permiten que los actores colectivos reinventen sus roles y estrategias para el acceso a la información. Por el otro, que las grandes ONG se han consolidado como grandes concentradoras de recursos económicos, razón por la cual tienen tanta capacidad de producción de contenidos. En este sentido, Human Rights Watch, por ejemplo, tiene "una fuerza laboral que rivaliza con todas las agencias de noticias internacionales de los principales proveedores de noticias de Estados Unidos como el New York Times o el Washington Post” (Powers, 2016a, p. 318); mientras que la propia Amnistía Internacional “emplea a más de 125 investigadores para recopilar información sobre temas de derechos humanos en todo el mundo” (Powers, 2016a, p. 318).

Sin duda, las nuevas tecnologías están incidiendo directamente en la relación entre derechos humanos y comunicación, transformando algunas de las estrategias del activismo internacional en derechos humanos (Tumber \& Waisbord, 2017). Sin embargo, también es claro que las organizaciones no gubernamentales que impulsan estas costosas campañas son las principales organizaciones internacionales, que tienen fuertes vínculos con organismos intergubernamentales, agencias de financiación, entre otras, reproduciendo igualmente un sesgo de selección y atención de los problemas que cubren.

Dentro de la misma línea que se pregunta por el rol de las ONG como productoras de la información, los trabajos de McPherson $(2016,2012)$ sostienen que la relación entre las organizaciones de noticias, medios de comunicación y las ONG de derechos humanos se presenta en un doble sentido. Por una parte, la información que producen las ONG puede generar un "subsidio de información" para los periodistas, dado que las ONG generan información valiosa sobre temas problemáticos difíciles de cubrir. Al mismo tiempo, en la 
medida que la credibilidad de la fuente es fundamental para la lógica del periodismo, las ONG con mayor reputación proporcionan materiales que cubren los parámetros de credibilidad y autorización, generando igualmente un "subsidio de verificación" que en todos los casos disminuye el costo de la investigación y verificación de los hechos.

Este costo de verificación “cubierto” por el trabajo de las ONG se fundamenta, principalmente, en el hecho de que la información y documentación que estas organizaciones realizan están pensadas para generar procesos no sólo de presión pública, sino también para apoyar demandas legales y mecanismos de rendición de cuentas, por lo cual la revisión editorial de la información de las ONG más profesionalizadas suele ser incluso más rigurosa que la de las editoriales de prensa (Pruce \& Cosima, 2016, p. 413). De esta manera, la interrelación entre ONG y medios se vuelve cada vez más importante, dice McPherson (2016), dado que los modelos de negocio de los medios de comunicación y periodísticos tradicionales encuentran mayores problemas de financiamiento, lo que los obliga a realizar recortes de personal. Nuevamente, el caso de Human Rights Watch es destacado, organización que ya tiene sedes en lugares como Beirut, Nairobi y Tokio, asumiendo un rol activo en la política de producir los propios contenidos de información a las secciones de prensa internacional (Powers, 2018).

\section{El sesgo internacional de la literatura}

Como hemos apreciado, el campo de estudio de la relación entre ONG de derechos humanos y medios de comunicación se distingue por el reconocimiento de la política de la información como estrategia clave del activismo en defensa de los derechos humanos. Esta relación nos habla, justamente, de la forma en que la información generada para visibilizar violaciones de derechos humanos y cuestionar los abusos de poder, puede circular y tener difusión entre amplios públicos. Así, hemos identificado dos grandes ejes en los que se divide el énfasis dado a esta relación: por un lado, aquellos que entienden a los medios de comunicación como mediadores de la política de la información; por otro lado, quienes aceptando lo anterior, se preguntan por el rol especial de las ONG en el proceso mismo de producción y generación de la información, su relación con las nuevas tecnologías y la forma como las organizaciones se convierten en productoras creíbles de contenido para los propios medios.

Como se sintetiza en la Tabla 1, las líneas de investigación y perspectivas analíticas han dado insumos importantes. En este sentido, las investigaciones son claras en mostrar que sería impensable un proceso de presión y cuestionamiento del abuso del poder sin la interacción estratégica entre ONG y medios de comunicación para la circulación y difusión de información. Es igualmente claro que las estrategias de los actores colectivos para "narrar", "visibilizar" y generar empatía en torno a violaciones de derechos humanos están igualmente condicionadas, por lo menos en cuanto a la circulación en medios, por las políticas editoriales y la tendencia dominante de prestarle atención a violaciones de derechos civiles y políticos. 
Política de la información, las ong y los medios de comunicación en los conflictos por derechos humanos: un balance

Tabla 1. Principales ejes de análisis de la relación ONG de derechos humanos y medios de comunicación.

\begin{tabular}{|c|c|c|}
\hline & \multicolumn{2}{|c|}{ Política de la información } \\
\hline & Medios como mediadores & $\begin{array}{l}\text { ONG como productoras de } \\
\text { información para los medios }\end{array}$ \\
\hline $\begin{array}{l}\text { Principales } \\
\text { preguntas }\end{array}$ & $\begin{array}{l}\text { El rol que juegan los medios en } \\
\text { la política de la información y } \\
\text { los factores que permiten que } \\
\text { las ONG tengan incidencia en } \\
\text { la producción de noticias en los } \\
\text { medios de comunicación. }\end{array}$ & $\begin{array}{l}\text { El rol que juegan las ONG, dentro de la } \\
\text { dinámica de las nuevas tecnologías, en } \\
\text { la producción directa de información } \\
\text { para las secciones de información } \\
\text { internacional de los medios de } \\
\text { comunicación. }\end{array}$ \\
\hline Ejes de análisis & $\begin{array}{l}\text {-Qué estrategias siguen las ONG } \\
\text { para incidir en el contenido de los } \\
\text { medios. } \\
\text {-Qué tipo de narrativas son más } \\
\text { proclives a recibir atención de los } \\
\text { medios y qué implicaciones tiene } \\
\text { el tipo de noticias que los medios } \\
\text { cubren en materia de derechos } \\
\text { humanos. } \\
\text {-Cuáles y por qué ciertas ONG } \\
\text { tienen más capacidad de incidencia } \\
\text { en los medios que otras. }\end{array}$ & $\begin{array}{l}\text {-Los nuevos recursos con los que } \\
\text { cuentan las ONG para producir } \\
\text { información de campo en el área } \\
\text { internacional. } \\
\text {-Los efectos positivos o negativos del } \\
\text { rol activo de las ONG en la producción } \\
\text { de información para las secciones } \\
\text { internacionales de los medios. } \\
\text {-El impacto de las nuevas tecnologías } \\
\text { en el activismo y la producción de } \\
\text { información. }\end{array}$ \\
\hline $\begin{array}{l}\text { Objetos } \\
\text { empíricos }\end{array}$ & $\begin{array}{l}\text {-Contenido de prensa y medios. } \\
\text {-Estrategias de construcción de } \\
\text { información y narrativas de las } \\
\text { ONG. }\end{array}$ & $\begin{array}{l}\text {-Las prácticas de trabajo de las ONG } \\
\text { internacionales. }\end{array}$ \\
\hline
\end{tabular}

Fuente: elaboración propia.

Ahora bien, más allá de estos aportes, es necesario cuestionar lo que denomino un sesgo internacionalista y elitista en la literatura. Esto es, todas las perspectivas e investigaciones están abordando los casos de las grandes organizaciones de derechos humanos transnacionales y los grandes medios de comunicación internacionales. Lo anterior se ve reflejado en el hecho de que las investigaciones, en su gran mayoría, han analizado empíricamente la influencia que tienen grandes ONG como Human Rights Watch y Aministía Internacional en la producción de noticias e información en medios como CNN, The New York Times, entre otros, específicamente en las secciones de noticias internacionales. Lo anterior se debe a que la problematización de todos los trabajos sobre la relación entre medios de comunicación y ONG está orientada sobre el rol que cumplen las organizaciones en la cobertura e información sobre la "realidad internacional", de allí que uno de los ejes de mayor preocupación en los últimos años sea el de explicar cómo esta relación reduce costos para la prensa en el levantamiento de información en "el extranjero” (Powers, 2016b; 2018). 
Este énfasis en la dimensión internacional y en las grandes organizaciones no gubernamentales reproduce una lectura que define la socialización de los derechos humanos como una dinámica que va de los países centrales hacia la periferia (López, 2013; 2017; López e Hincapié, 2015). En la medida que las teorías de la socialización y activismo transnacional de derechos humanos fueron pensadas por las relaciones internacionales para explicar procesos de presión sobre gobiernos autoritarios dictatoriales, la investigación sobre la política de la información se centró en cómo se hacían visibles internacionalmente las situaciones y violaciones que los gobiernos autoritarios tenían "bloqueados" a nivel nacional. Como es de esperarse, entonces, estas perspectivas encuentran serias limitaciones cuando el estudio demanda entender procesos donde la defensa de los derechos humanos se inscribe en lógicas de regímenes con cierto grado de pluralismo electoral, en los cuales cierto tipo de libertades son respetadas, pero "paradójicamente" persisten graves violaciones de derechos humanos.

La lógica de denuncia pública sobre "claras" violaciones de derechos humanos por gobiernos dictatoriales no es la que prevalece hoy, por el contrario, persisten contextos donde los gobiernos "retóricamente" se comprometen con los derechos humanos ante la comunidad internacional, pero se reproducen las graves violaciones de derechos dentro de los Estados. En contextos de democracia formal, donde existe relativa circulación de la información y espacios de expresión, las disputas y conflictos por los derechos humanos se generan, precisamente, en los Estados, y el rol de la relación entre ONG y medios de comunicación estatales se vuelve central.

Finalmente, dado que las preguntas de la literatura académica están orientadas a la influencia que las grandes ONG de derechos humanos tienen en los grandes medios de comunicación para visibilizar las "situaciones internacionales", es inexistente el cuestionamiento sobre: a) los campos de relaciones en que se insertan las ONG dentro de los Estados; y b) las diferentes estructuras de los sistemas de medios de comunicación que pueden determinar, igualmente, la relación que las ONG puedan establecer con los medios. Esta falta de problematización tiene implicaciones en el análisis en la medida que no se profundiza en los contextos, cuáles, cuántas y porqué ciertas ONG son consultadas y tienen repercusiones mediáticas en relación con los amplios campos de acción colectiva de defensa de derechos humanos (pensando en colectivos de víctimas y organizaciones con menos recursos). De la misma manera, sin cuestionar la propia estructura del sistema de medios de comunicación no podremos entender por qué la información circula o no, y las implicaciones que esto tiene para reforzar las situaciones de abuso del poder en aparentes contextos de apertura democrática. 
Política de la información, las ong y los medios de comunicación en los conflictos por derechos humanos: un balance

\section{Conclusiones}

En este artículo he realizado un balance crítico de la literatura académica que estudia la relación entre ONG de derechos humanos y medios de comunicación. El principal eje analítico que ordena los estudios es el reconocimiento de la política de la información como principal repertorio de activismo por los derechos humanos, proceso en el que la relación que se establece entre ONG y medios en la circulación de la información se vuelve central. Una vez definido este marco general en el que se inscriben las investigaciones, identifiqué dos énfasis, por un lado aquel concentrado en la capacidad y factores que permiten que las ONG incidan en la producción de noticias e información en los medios de comunicación; por otro lado, aquella que se pregunta, principalmente, por las implicaciones que tiene el que las ONG asuman, cada vez más, roles de productoras directas de información para los medios, reconocimiento los cambios tecnológicos y las lógicas de cubrimiento que siguen estos actores colectivos.

En la medida que las dinámicas sociopolíticas en el mundo se han transformado (y especialmente en América Latina con la consolidación de regímenes híbridos), es cada vez mayor el consenso en torno a la importancia de la dinámica contenciosa dentro de los Estados y la manera en que los actores colectivos apelan a diferentes repertorios para la defensa de los derechos humanos (Dancy \& Sikkink, 2017; López, 2017; López e Hincapié, 2015; Tsutsui y Smith, 2018). Por estas razones, he realizado una crítica a la literatura dado sus sesgos de análisis en la dinámica internacional y en el trabajo de las grandes ONG de derechos humanos, lo que lleva a invisibilizar los múltiples procesos y conflictos por derechos humanos que se generan en los Estados, el trabajo de múltiples y diversas organizaciones que bajo parámetros de democracia formal adelantan esfuerzos por generar procesos de control al abuso del poder.

Son múltiples las preguntas que esta agenda de investigación demanda. ¿La política de información ha permitido mayor rendición de cuentas? ¿Cuál ha sido el rol de los sistemas de medios y prensa en el cubrimiento y la forma como retoman la información de derechos humanos? ¿Las ONG y actores colectivos se han posicionado en los medios, y el campo de derechos humanos en general para influir en los marcos con que se cubren los conflictos y las crisis? ¿Podemos pensar en un proceso de socialización de los derechos humanos en los medios al interior de los estados? Todas estas preguntas son más necesarias si tenemos en cuenta que en escenarios como los latinoamericanos, muchas veces coexisten sistemas de medios altamente concentrados, los principales medios tienen relaciones estrechas con el poder político, existen políticas de restricción a la cobertura de la violencia y crisis de derechos humanos, aunado al aumento exponencial de la violencia que experimentan aquellos periodistas y activistas críticos que salen de dicha lógica. 


\section{Referencias}

Álvarez, S. (2009). Beyond NGO-ization: Reflections from Latin America. Development, 52 (2), 175-184.

Becker, J. (2013). Campaigning for Justice: Human Rights Advocacy in Practice. Stanford, CA: Stanford University Press.

Bennett, L. \& Segerberg, A. (2012). The Logic of Connective Action. Information, Communication \&Society, 15 (5), 739-768.

Bob, C. (2002). Merchants of Morality. Foreign Policy, 129, 36-45.

Bob, C. (2005). The Marketing of Rebellion: Insurgents, Media and International Activism. Nueva York: Cambridge University Press.

Bob, C. (2010). The Market for Human Rights. En A. Prakash. y M. Gugerty. (Eds.), Advocacy Organizations and Collective Action (pp. 133-154). Cambridge: Cambridge University Press.

Borer, T. (Ed.). (2012). Media, Mobilization, and Human Rights: Mediating Suffering. Nueva York: Zed Books.

Brysk, A. (1994). The politics of Human Rights in Argentina. California: Stanford University Press.

Brysk, A. (2009). Communicative action and human rights in Colombia. When words fail. Colombia Internacional, 69, 36-49.

Brysk, A. (2013). Speaking Rights to Power. Constructing Political Will. Nueva York: Oxford University Press.

Brysk, A. \& Shafir, G. (2007). National Insecurity and Human Rights: Democracies Debate Counterterrorism. California: University of California Press.

Burgerman, S. (2001). Moral victories. How activists provoke multilateral action. Ithaca: Cornell University Press.

Cárdenas, S. (2007). Conflict and Compliance State Responses to International Human Rights Pressure. Philadelphia: University of Pennsylvania Press.

Cárdenas, S. (2010). Human Rights in Latin America. A politics of terror and hope. Philadelphia: University of Pennsylvania Press.

Carpenter, C. (2014). Lost Causes:Agenda-Vetting in Global Issue Networks and the Shaping of Human Security. Ithaca: Cornell University Press.

Carpenter, C. (2007a). Setting the Advocacy Agenda: Theorizing Issue Emergence and Nonemergence in Transnational Advocacy Networks. International Studies Quarterly, 51, 99-120. 
Política de la información, las ong y los medios de comunicación en los conflictos por derechos humanos: un balance

Carpenter, C. (2007b). Studying Issue (Non)-Adoption in Transnational Networks. International Organization, 61 (3), 643-667.

Castells, M. (2011). Comunicación y poder. Madrid, España: Alianza.

Chadwick, A. (2013). The Hybrid Media System: Politics and Power. Oxford: Oxford University Press.

Clark, A. M. (2001). Diplomacy of Conscience: Amnesty International and Changing Human Rights Norms. Princeton, NJ: Princeton University Press.

Cottle, S. \& Nolan, D. (2007). Global humanitarianism and the changing aid-media field. Journalism Studies, 8 (6), 862-878.

Dancy, G. \& Sikkink, K. (2017). Human Rights Data, Processes, and Outcomes: How Recent Research Points to a Better Future. In S. Hopgood., J. Snyder. y L. Vinjamuri. (Eds.), Human Rights Futures (pp. 24-59). Cambridge: Cambridge University Press.

Dogra, N. (2014). Representations of Global Poverty: Aid, Development and International NGOs. London: Tauris.

Dupuy, K., Ron, J. \& Prakash, A. (2016). Hands Off My Regime! Governments' Restrictions on Foreign Aid to Non-Governmental Organizations in Poor and Middle-Income Countries. World Development, 84, 299-311.

Fenton, N. (2010). NGOs, New Media and the Mainstream News. In N. Fenton. (Ed.), New Media, Old News (pp. 153-168). London: Sage.

Franklin, J. (2015). Human Rights Naming and Shaming: International and Domestic Processes. In H. Friman. (Ed.), The Politics of Leverage in International Relations: Name, Shame and Sanction (pp. 43-60). London: Palgrave Macmillan.

Freedom House. (2018). Freedom in the world. 2018. Recovered from https: / / freedomhouse. $\mathrm{org} /$ sites/default/files/FH_FITW_Report_2018_Final_SinglePage.pdf.

Gordon, N. (2008). Human Rights, Social Space and Power: Why Do some NGOS exert more Influence than Others? International Journal of Human Rights, 12 (1), 23-39.

Gordon, N. (2014). Human Rights as a Security Threat: Lawfare and the Campaign against Human Rights NGOs. Law \& Society Review, 48 (2), 311-344.

Hafner-Burton, E. (2008). Sticks and Stones: Naming and Shaming the Human Rights Enforcement Problem. International Organization, 4 (62), 689-716.

Hagan, M. (2010). The human rights repertorie: its strategic logic, expectations and tactics. International Journal of Human Rights, 14 (4), 559-583.

Hincapié, S. (2015). Estado y derechos humanos en México: claves analíticas para su interpretación. El Cotidiano, 30 (194), 89-96. 
Hincapié, S. (2017). Acción colectiva de las mujeres y derechos humanos en México. Movilizando el dolor en medio del conflicto armado. Estudios Socio-Jurídicos, 19 (2), 97 127.

Hopgood, S. (2006). Keepers of the Flame: Understanding Amnesty International. Ithaca, NY: Cornell University Press.

Jetschke, A. (2010). Human Rights and State Security: Indonesia and the Philippines. Philadelphia: University of Pennsylvania Press.

Jetschke, A. \& Liese, A. (2013). The power of human rights a decade after: from euphoria to contestation? En T. Risse., S. Ropp. \& K. Sikkink. (Eds.), The persistent power of human rights. From commitment to compliance (pp. 26-42). Cambridge: Cambridge University Press.

Keck, M. \& Sikkink, K. (2000). Activistas sin fronteras. Redes de defensa en politica internacional. Ciudad México, México: Siglo XXI.

López, J.A. (2013). Los derechos humanos en movimiento: una revisión teórica contemporánea. Espiral. Estudios sobre Estado y Sociedad, 56, 9-37.

López, J. A. (2017). Movilización y acción colectiva por los derechos humanos en la paradoja de la institucionalización. Estudios Políticos, 51, 57-78.

López, J. A. e Hincapié, S. (2015). Campos de acción colectiva y ONG de derechos humanos. Herramientas teóricas para su análisis. Sociológica, 30 (85), 9-37.

McPherson, E. (2012). How editors choose which human rights news to cover: a case study of Mexican newspapers. In T. Borer. (Ed), Media, mobilization and human rights: mediating suffering (pp. 96-121). London: Zed.

McPherson, E. (2016). Source credibility as "information subsidy": Strategies for successful NGO journalism at Mexican human rights NGO. Journal of Human Rights, 15 (3), 330-346.

Menon, G. (2009). Genesis and role of NGOs in protecting human rights. New Delhi: Dominant Publisher and Distributors.

Murdie, A. \& David, D. (2011). Shaming and Blaming: Using Events Data to Assess the Impact of Human Rights INGOs. International Studies Quarterly, 1 (56), 1-16.

Nash, K. (2009). The Cultural Politics of Human Rights. Cambridge: Cambridge University Press.

Olvera, A. (1999). La sociedad civil. De la teoría a la realidad. Ciudad de México, México: El Colegio de México.

Orgad, S. (2013). Visualizers of Solidarity: Organizational Politics in Humanitarian and International Development NGOs. Visual Communication, 12 (3), 295-314.

Powers, M. (2016a). A new era of human rights news? Contrasting two paradigms of human rights news-making. Journal of Human Rights, 15 (3), 314-329. 
Política de la información, las ong y los medios de comunicación en los conflictos por derechos humanos: un balance

Powers, M. (2016b). Beyond Boon or Bane. Using normative theories to evaluate the newsmaking efforts of NGOs. Journalism Studies, 18 (9), 1070-1086.

Powers, M. (2016c). Opening the news gates? Humanitarian and human rights NGOs in the US news media, 1990-2010. Media, Culture \& Society, 38 (3), 315-331.

Powers, M. (2015). Contemporany NGO-Journalist Relations: Reviewing and Evaluating an Emergent Area of Research. Sociology Compass, 9 (6), 427-437.

Powers, M. (2018). NGOs as Newsmakers:The Changing Landscape of International News. Columbia: Columbia University Press.

Pruce, J. (Ed.). (2015). The Social Practice of Human Rights. Nueva York: Palgrave Macmillan.

Pruce, J. \& Cosima, A. (2016). Beyond naming and shaming: New modalities of information politics in human rights. Journal of Human Rights, 15 (3), 408-425.

Risse, T., Ropp, S. \& Sikkink, K. (1999). The Power of Human Rights: International Norms and Domestic Change. Cambridge: Cambridge University Press.

Risse, T., Ropp, S. \& Sikkink, K. (2013). The Persistent Power of Human Rights: From Commitment to Compliance. Cambridge: Cambridge University.

Ristovska, S. (2016). The rise of eyewitness video and its implications for human rights: Conceptual and methodological approaches. Journal of Human Rights, 15 (3), 347-360.

Ron, J., Ramos, H. \& Rodgers, K. (2005). Transnational information politics: NGO human rights reporting, 1986-2000. International Studies Quarterly, 49 (3), 557-588.

Rosanvallon, P. (2007). La contrademocracia: la política en la era de la desconfianza. Buenos Aires, Argentina: Manantial.

Rothmyer, K. (2011). Hiding the Real Africa:Why NGOs Prefer Bad News. Columbia Journalism Review. Recovered from https://archives.cjr.org/reports/hiding_the_real_africa.php.

Seaga, I. (2012). Human Rights Journalism:Advances in Reporting Distant Humanitarian Interventions. London: Palgrave Macmillan.

Shor, E. (2008). Conflict, Terrorism and the Socialization of Human Rights Norms: The Spiral Model Revisited. Social Problems, 55 (1), 117-138.

Simmons, B. (2009). Mobilizing for Human Rights: International Law in Domestic Politics. Cambridge: Cambridge University Press.

Stammers, N. (2009). Human Rights and Social Movements. London: Pluto.

Tarrow, S. (2005). The new transnational activism. Cambridge: Cambridge University Press.

Thompson, J. (2001). El escándalo político. Poder y visibilidad en la era de los medios de comunicación. Barcelona, España: Paidós. 
Thrall, T., Stecula, D. \& Sweet, D. (2014). May we have your attention please?: Human rights NGOs and the problem of global communication. International Journal of Press/Politics, 19 (2), 135-159.

Tsutsui, K. \& Smith, J. (2018). Human Rights and Social Movements: From the Boomerang Pattern to a Sandwich Effect. In D. Snow, S. Soule, H. Kriesi \& H. McCammon. (Eds.), TheWiley Blackwell Companion to Social Movements (pp. 586-601). London: Wiley Blackwell.

Tsutsui, K., Whitlinger, C. \& Alwyn, L. (2012). International Human Rights Law and Social Movements: States' Resistance and Civil Society's Insistence. Annual Review of Law and Social Science, 8, 367-396.

Tumber, H. \& Waisbord, S. (2017). The Routledge Companion to Media and Human Rights. Basingstoke: Taylor \& Francis.

Tumber, H. \& Waisbord, S. (2004). Political Scandals and Media Across Democracies. American Behavioral Scientist, 47 (8), 1031-1039.

Waisbord, S. (2011). Can NGOs Change the News? International Journal of Communication, 5 , 142-165.

Waisbord, S. (2016). Media Movements: Civil Society and Media Policy Reform in Latin Americas. London: Zed Books.

Winston, M. (2001). Assessing the effectiveness of human rights NGOs: Amnesty International. En C.E. Welch. (Ed.), NGOs and Human Rights: Promise and Performance. Philadelphia: University of Pennsylvania Press.

Winston, M. \& Pollock, J. (2016). Human rights in the news: Balancing new media participation with the authority of journalists and human rights professionals. Journal of Human Rights, 15 (3), 307-313.

Wotipka, C. \& Tsutsui, K. (2008). Global human rights and State sovereignty: State ratification of International Human Rights Treaties, 1965-2001. Sociological Forum, 23 (4), 724-754. 